\title{
Optimum use of a 4-element Yagi-Uda Antenna for the Reception of Several UHF TV Channels
}

\author{
C.J.S.A.H. Perera
}

\begin{abstract}
Outdoor Yagi-Uda antennas are frequently used in Sri Lanka for the reception of UHF and VHF TV channels. An antenna designed for a particular channel can show poor reception characteristics to other TV channels due to their operating frequencies and physical locations. The first few TV stations, which were set up in nineteen eighties, had VHF transmissions. Those TV channels still operate on VHF, while some of them supplement with UHF transmissions. A large number of TV channels in Sri Lanka use UHF channels for the transmission of their TV programs. The paper studies the dependence of the maximum gain of the antenna, the input power to the dipole of the antenna, and the radiation pattern of the antenna on the operating frequency which are the key indicators of its receiving characteristics.

Considering the physical distribution of TV stations in the lowlands of Sri Lanka, this paper searches ways of optimizing reception characteristics of a 4-element Yagi-Uda UHF antenna.
\end{abstract}

Keywords: Yagi-Uda array, radiation pattern, antenna gain

\section{Introduction}

The Yagi-Uda antenna was first proposed by Prof. Shintaro Uda and Prof. Hidetsugu Yagi in 1926. A considerable improvement in the gain and the directivity of the antenna could be achieved through this antenna. The antenna was frequently used in radar applications during Second World War.

With the introduction of TV broadcasting, the Yagi-Uda antenna became very popular among TV viewers, mainly due to its high gain. As the number of elements in it is increased, its gain and directivity also increase making it ideal to receive a distant TV station.

A good study on the dependence of the directivity and the beamwidth of the antenna on its number of elements has been done in [6]. In the calculation of the gain, directivity and beamwidth of a Yagi-Uda array, the current distributions in the driving- and the driven elements play an important role. Although the sinusoidal current distribution is a good approximation for element lengths around $\lambda / 2$, it is not so for elements of higher lengths.

This issue has been discussed in [1]. In this paper, the author discusses how gain optimization could be achieved for dipole lengths of $\lambda$ and $1.5 \lambda$ using a modified nonsinusoidal current distribution.

When there are several TV channels, the viewers always prefer to use the same antenna for the reception of all the channels, even though it may not be practically possible. In order to design an antenna to cover several TV channels, not only the gain but also the beamwidth of the antenna has to be considered. To find out whether a given antenna can receive a remote TV station, one should know the $3 \mathrm{~dB}$ bandwidth (say $\alpha$ ) of the antenna. With the help of two circular arcs with inscribed angles $\alpha$ and having the two stations as the two common end points, the antenna coverage can easily be predicted.

\section{Theoretical Background}

2.1 Coverage of more than one Station with a Single Antenna

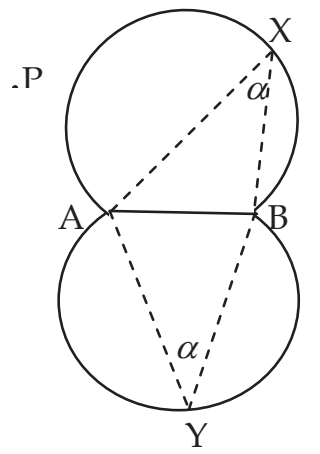

Two equal circular arcs with inscribed angle $\alpha$.

A, B - TV stations $\alpha$ - beamwidth

\section{Figure 1 - Antenna Coverage for two stations}

The antenna located at Point $\mathrm{P}$ outside the two arcs $\mathrm{AXB}$ and $\mathrm{AYB}$ should receive both TV channels without any problem, provided that the receiving antenna has approximately the same $\alpha$ for the operating frequencies of the two transmitting stations. Here, the transmissions of the antennas are assumed to be isotropic.

Eng. C.J.S.A.H.Perera, BSc. Eng. (Hons.)(Moratuwa), MSc(Munich), CEng. Senior Lecturer, Department of Electrical and Computer Engineering, Open University of Sri Lanka Email:cjper@ou.ac.lk 


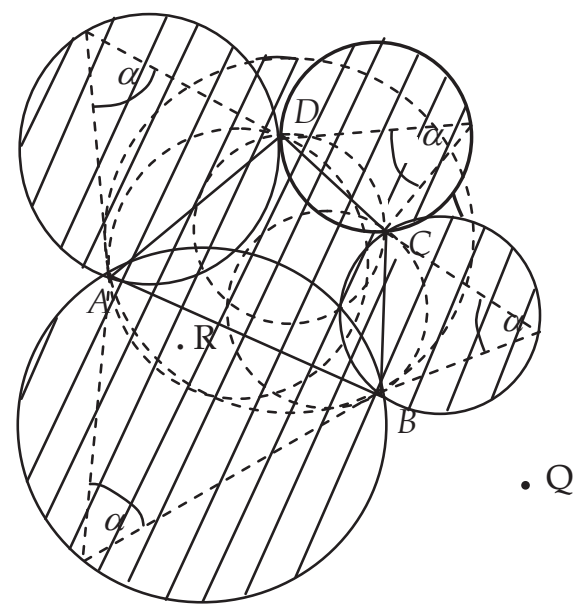

Figure 2 - Reception of multiple stations

When there are 4 transmitting stations A, B, C, and $D$, Point $Q$ outside the dark area should receive all 4 stations trouble free.

\subsection{Antenna Equations}

A very good analysis of the Yagi-Uda array with necessary fundamental equations is given in [3].

Using Maxwell's equations, the equations for scalar potential $\varphi$ and vector potential $A$ can be derived with the help of Gauge Transformation and Lorentz conditions. In order to calculate electric- and magnetic field components,

$E_{Z}(z, \rho), \quad E_{\rho}(z, \rho)$ and $H_{\varphi}(z, \rho)$ shown in

Figure 3 , it is sufficient to know $A_{z}(z, \rho)$, the

$z$-component of $A . A_{Z}(z, \rho)$ is given by $A_{Z}(z, \rho)=\frac{\mu}{4 \pi} \int_{-h}^{h} I\left(z^{\prime}\right) \frac{e^{-j k R}}{R} d z^{\prime}$,

where $R=\sqrt{\rho^{2}+\left(z-z^{\prime}\right)^{2}}$

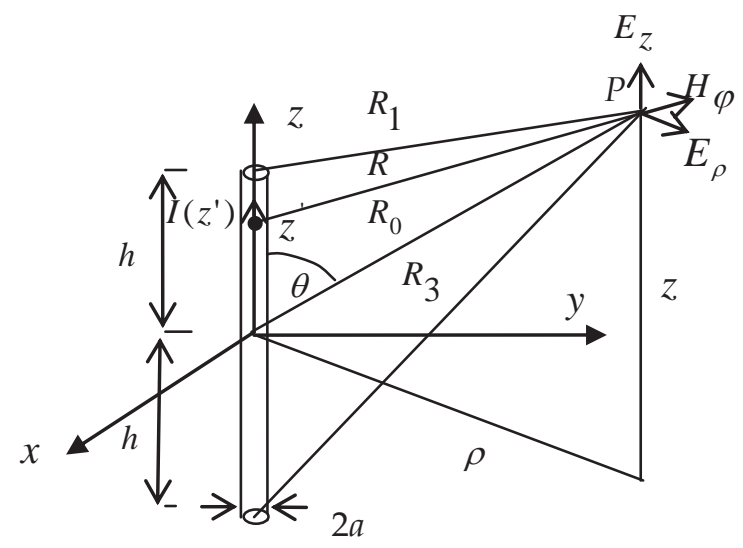

Figure 3 - Field components due to a dipole antenna located at a distant Point $P$
With the assumption that the antenna current $I(z)$ is distributed sinusoidally, $I(z)$ can be written as:

$$
I(z)=I_{0} \frac{\sin (k(h-|z|))}{\sin k h}=I_{m} \sin (k(h-|z|))
$$

For a half wave dipole, $I(z)$ reduces to

$I_{0} \cos (k z)$ since $k h=\pi / 2$

Using the relationship,

$j \omega \mu E_{Z}(z, \rho)=\partial_{z}{ }^{2} A_{z}+k^{2} A_{z}$ in Equation (1)

$E_{Z}(z, \rho)$ can be derived as

$E_{Z}(z, \rho)=-\frac{j \eta I_{m}}{4 \pi}\left[\frac{e^{-j k R_{1}}}{R_{1}}+\frac{e^{-j k R_{2}}}{R_{2}}-2 \cos (k h) \frac{e^{-j k R_{0}}}{R_{0}}\right]$

In a similar manner, he field components

$E_{\rho}(z, \rho)$ and $H_{\varphi}(z, \rho)$ can be derived.

$H_{\varphi}(z, \rho)=\frac{j I_{m}}{4 \pi \rho}\left[e^{-j k R_{1}}+e^{-j k R_{2}}-2 \cos (k h) e^{-j k R_{0}}\right]$

$$
\begin{aligned}
E_{\rho}(z, \rho) & =\frac{j I_{m}}{4 \pi \rho}\left[\frac{z-h}{R_{1}} e^{-j k R_{1}}+\frac{z+h}{R_{2}} e^{-j k R_{2}}\right. \\
& \left.-2 \cos (k h) \frac{z}{R_{0}} e^{-j k R_{0}}\right]
\end{aligned}
$$

$E_{Z}(z, \rho)$ can be used to calculate the mutual impedance $Z_{21}$ induced by the above dipole (say dipole 1) on another dipole (say dipole 2).

In an antenna array consisting of $K$ elements, the mutual impedance $Z_{p q}$ on the $p^{\text {th }}$ element due to the $q^{\text {th }}$ element can be calculated from the $E$ field strength $E_{p q}(z)$ at $p^{\text {th }}$ element due to $q^{\text {th }}$ element:

$$
Z_{p q}=-\frac{1}{I_{p} I_{q}}-\int_{h_{p}}^{h_{p}} E_{p q}(z) I p(z) d z
$$

$E_{p q}(z)$ can be calculated using Equation (3).

$I_{p}$ - Current in $p^{\text {th }}$ element, $I_{q}$ - Current in $q^{\text {th }}$ element, $2 h_{p}$ - Length of $p^{\text {th }}$ element.

When $p=q, Z_{p q}$ becomes the self-impedance of the $p^{\text {th }}$ element. This procedure can be 
extended to calculate the mutual impedance and the self-impedance of all elements in the array. Finally, the impedance matrix of the array $[Z]_{k \times k}$ given below consisting of selfimpedances and mutual impedances can be derived .

$[Z]=\left[\begin{array}{cccc}Z_{11} & Z_{12} & \ldots & Z_{1 k} \\ Z_{21} & Z_{22} & \ldots & Z_{2 k} \\ \cdot & \cdot & \ldots & \cdot \\ Z_{k 1} & Z_{k 2} & \ldots & Z_{k k}\end{array}\right]$, a matrix with

diagonal symmetry $Z_{p q}=Z_{q p}$

Since the voltage across the $p^{\text {th }}$ element $V_{p}$ consists of the self-induced voltage due to $Z_{p p}$ and the induced voltages due to rest of the $k-1$ elements , $V_{p}$ can be written as

$V_{p}=\sum_{q=1}^{k} Z_{p q} I_{q}, p=1,2, \ldots, k$

Therefore, the voltage vector for the $k$ elements can be calculated using the matrix equation

$\left[\begin{array}{c}V_{1} \\ V_{2} \\ \cdot \\ V_{k}\end{array}\right]=\left[\begin{array}{cccc}Z_{11} & Z_{12} & \ldots & Z_{1 k} \\ Z_{21} & Z_{22} & \ldots & Z_{2 k} \\ \cdot & \cdot & \ldots & \cdot \\ Z_{k 1} & Z_{k 2} & \ldots & Z_{k k}\end{array}\right]\left[\begin{array}{c}I_{1} \\ I_{2} \\ \cdot \\ I_{k}\end{array}\right]$

For a Yagi-Uda array consisting of a reflector, a dipole, and $k$-2 directors, all the voltages in the vector except $V_{2}$ become zero. If $V_{2}$ is set to 1 unit, the voltage vector can be written as $[V]=[0,1,0, \ldots 0]^{T}$, so that the currents in the elements $I_{1}, I_{2}, \ldots, I_{k}$ can be calculated from $[I]=[Z]^{-1}[V]$.

If a sinusoidal distribution for the antenna current $I(z)$ is assumed, the normalized gain of the array $g(\theta, \varphi)$ can be written as

$g(\theta, \varphi)=\left|\sum_{p=1}^{k} I_{p} \frac{\cos \left(k h_{p} \cos \theta\right)-\cos k h_{p}}{\sin k h_{p} \sin \theta} e^{j k x_{p} \sin \theta \cos \varphi}\right|^{2}$

where $\theta$ and $\varphi$ are the elevation angle and the azimuth angle respectively. [3]

The current values obtained from Equation (10) can be substituted in the equation for $g(\theta, \varphi)$ to calculate azimuthal- and polar gains.

\section{Experimental Setup}

The antenna equations mentioned above can be used to calculate the maximum directive gain of the antenna and the power input to the dipole using tools developed in [3] along with standard Matlab tools. The variation of the antenna gain $g(\theta, \varphi)$ in the horizontal plane and the radiation pattern of the antenna can also be calculated for the array shown in Figure 5 .

If the antenna has been designed for a frequency $f_{0}$, the corresponding maximum gain $G_{0}$ and the real part of the dipole current $I_{0}$ can be calculated. Since the dipole voltage $\left(V_{2}\right)$ is kept constant at 1 unit, $I_{0}$ is a measure of the power $P_{0}$ delivered to the dipole.

Therefore, the performance of the antenna at a frequency $f=f_{0}+\Delta f$ (at off resonance) is a function of the new gain $G_{f}$ in the desired direction, the real part of the dipole current $I_{f}$ and the reflection coefficient $\Gamma$ between the antenna and the TV receiver.

In grouping the transmitting stations, stations situated far away have been omitted.

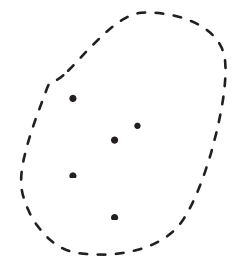

Group 1

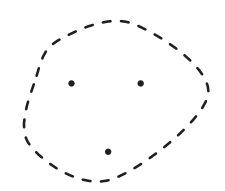

Group 2

\section{Figure 4 - Grouping of transmitting stations}

The possibility of receiving any other station without using the main lobe of the radiation pattern when the receiving antenna lies within the coverage area of all the stations (e.g. Point $Q$ of Figure 2) was also investigated.

If the antenna is located among the transmitting stations (e.g. Point R of Figure 2), then the group has to be subdivided as shown in Figure 4 to suit the total radiation pattern. 
A 4-element Yagi antenna was designed using the following parameters:

$$
\left|L_{1} L_{2} L_{3}\right| L_{3}
$$

Figure 5 - 4-element Yagi antenna

Frequency of operation $=f_{0}$;

Length of the dipole $\left(L_{2}\right)=0.48 \lambda_{0}$;

Length of the reflector $\left(L_{1}\right)=0.51 \lambda_{0}$;

Length of the first director $\left(L_{3}\right)=0.47 \lambda_{0}$;

Length of the second director $\left(L_{4}\right)=0.45 \lambda_{0}$;

Distance between the reflector and the dipole $=$ $0.25 \lambda_{0}$;

Distance between the first director and the dipole $=0.5 \lambda_{0}$;

Distance between the second director and the dipole $=\lambda_{0}$.

The behaviour of the antenna was investigated when there were other transmitting stations operating.

\section{Results and Observations}

The azimuthal gain plots for $\lambda / \lambda_{0}=0.83,1$ and 1.2 are shown in Figures 6(a), 6(b) and 6(c) respectively. Table 1 shows the dependence of beamwidth $\alpha$, dipole current $I_{f}$, and maximum azimuthal gain $G_{\max }$ on the frequency.

Table 1 - Variation of antenna characteristics with the operating frequency

\begin{tabular}{|c|c|c|c|c|}
\hline$f / f_{0}$ & $\lambda / \lambda_{0}$ & $\alpha$ & $I_{f} / I_{0}$ & $G_{\max } d B$ \\
\hline 1.2 & 0.83 & $90^{0}$ & 0.25 & 0 (back lobe) \\
\hline 1.18 & 0.85 & $90^{0}$ & 0.3 & 0 (back lobe) \\
\hline 1.15 & 0.87 & $40^{0}$ & 0.5 & $\begin{array}{c}0 \text { (back lobe) } \\
-1.5 \text { (front } \\
\text { lobe) }\end{array}$ \\
\hline 1.11 & 0.9 & $50^{0}$ & 1.8 & 0 \\
\hline 1.05 & 0.95 & $50^{0}$ & 1.2 & 0 \\
\hline 1 & 1 & $50^{0}$ & 1 & 0 \\
\hline 0.98 & 1.02 & $45^{0}$ & 1 & 0 \\
\hline 0.95 & 1.05 & $45^{0}$ & 0.9 & 0 \\
\hline 0.91 & 1.1 & $100^{0}$ & 0.6 & 0 \\
\hline 0.87 & 1.15 & $100^{0}$ & 0.4 & 0 \\
\hline 0.83 & 1.2 & $100^{0}$ & 0.3 & 0 \\
\hline
\end{tabular}

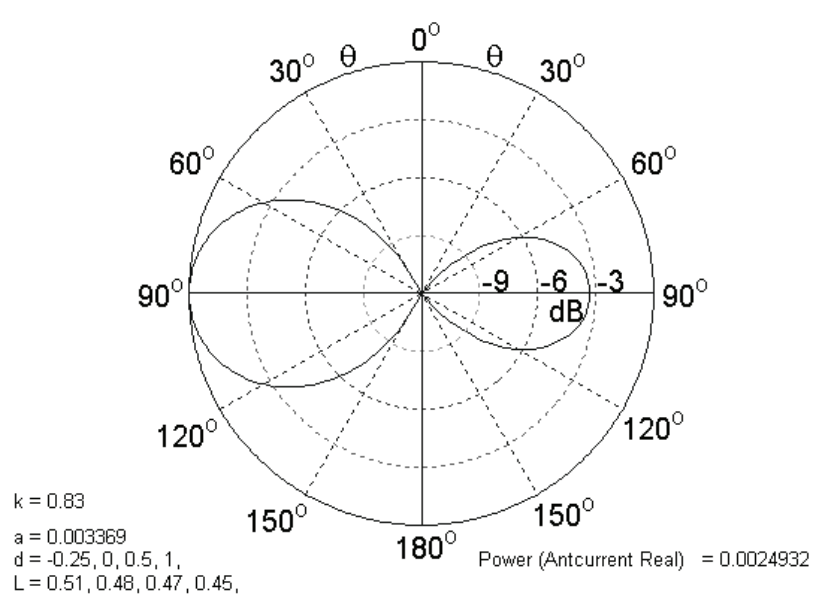

Figure 6(a) - Azimuthal gain plot

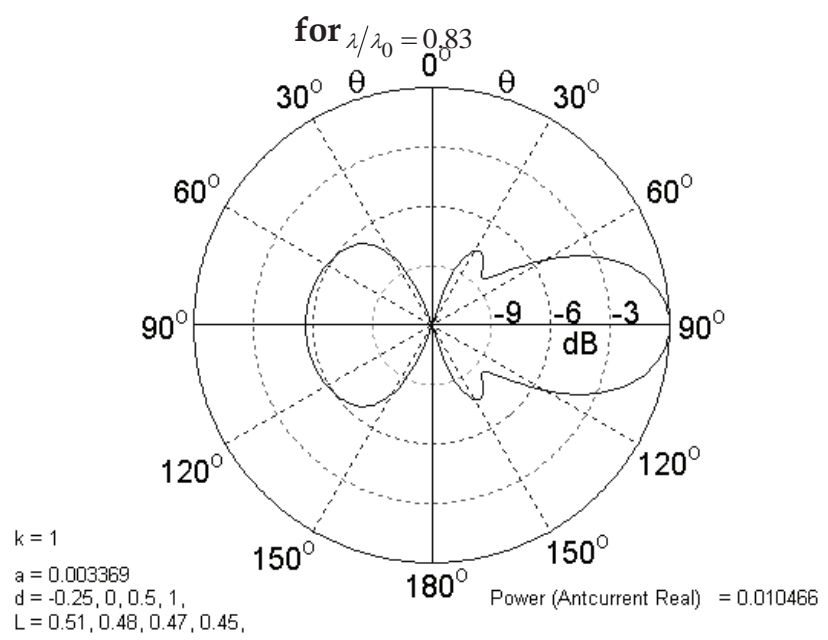

$L=0.51,0.48,0.47,0.45$,

Figure 6(b) - Azimuthal gain plot for $\lambda / \lambda_{0}=1$

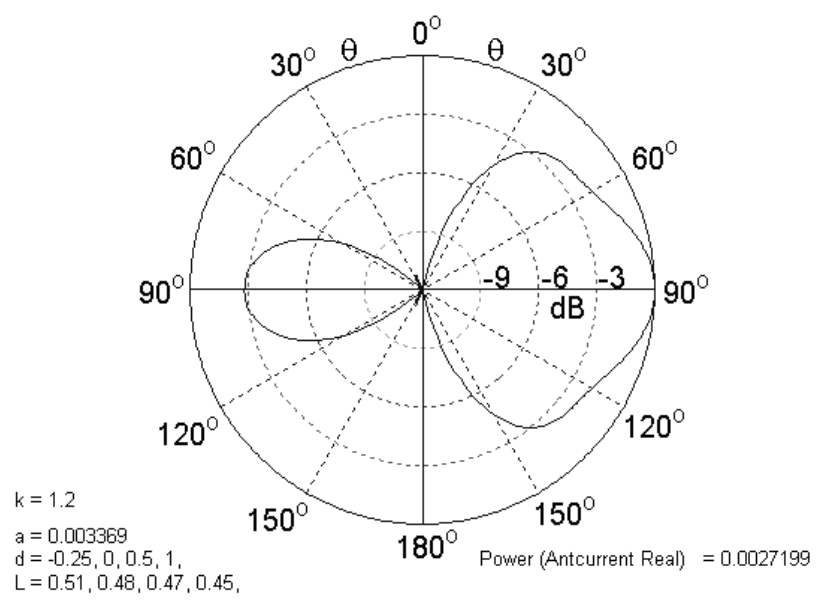

Figure 6(c) - Azimuthal gain plot for $\lambda / \lambda_{0}=1.2$

Variation of the radiation pattern of the antenna with the operating frequency shown in Figs. 6(a), 6(b) and 6(c) 
Two major groups of transmitting stations can be identified in the UHF TV network of Sri Lanka.

Group 1 - Transmitting stations located in the Colombo city as well as in close vicinity to it to cover Colombo district.

Group 2 - Transmitting stations located in the Ratnapura district (in Deniyaya and Gongala regions) to cover Matara district.

The locations of the UHF transmitting stations were marked on a map of Sri Lanka which has been drawn to an appropriate scale. This layout was used in all relevant geometrical calculations.

Group 1 consists of 5 stations. There are 3 stations in the city area, 1 station in the outskirts of the city and 1 station in the suburbs. Stations are located almost on a straight line.

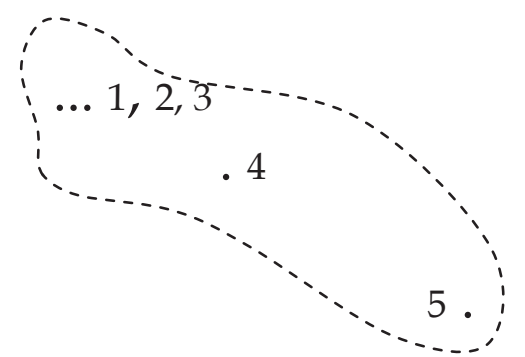

Figure 7 - Sub-grouping of Group 1

The antenna was designed for Station 1 (frequency $f_{1}=f_{0}, \lambda_{1}=\lambda_{0}$ ). The Stations 2, 3, 4 , and 5 correspond to $\lambda_{2}=0.92 \lambda_{0}$, $\lambda_{3}=1.17 \lambda_{0}, \quad \lambda_{4}=1.13 \lambda_{0}$ and $\lambda_{5}=1.02 \lambda_{0}$ respectively.

As per Table.1, the beamwidth variation of the antenna for the above mentioned frequency range is in the range $45^{\circ}-100^{\circ}$. The $I / I_{0}$ ratio drops to 0.4 and 0.5 for $\lambda_{3}$ and $\lambda_{4}$ respectively.

Thus, the antenna should be able to receive Stations 1, 2, and 5 clearly. A moderate level of reception can be expected for the Stations 3 and 4 due to the drop in the $I / I_{0}$ ratio.

If the line connecting 1 and 5 is taken as $\mathrm{AB}$ (Figure 1), two arcs can be drawn with $\alpha=45^{\circ}$. Signal reception outside the closed area generated by the two arcs should be satisfactory. This should cover the areas beyond Ragama, Balummahara, Weliveriya, Kaluaggala, Godagama, Homagama, Kahathuduwa, Pokunuwita and Panadura.
It appears that signal reception within the area of the group for all 5 stations would be somewhat difficult.

Since Station 4 has a very high beamwidth $\left(100^{\circ}\right)$,viewers in Kotte, Malabe, Dehiwala and Wellawatta areas should be able to receive it together with Stations 1, 2 and 3.

Group 2 consists of 5 stations. Three of the stations are located at one place (village) and the other 2 stations about $12 \mathrm{~km}$ away from it.

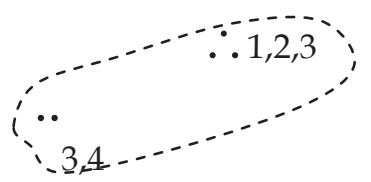

\section{Figure 8 - Sub-grouping of Group 2}

The antenna is designed for Station 1 (frequency $f_{1}=f_{0}, \quad \lambda_{1}=\lambda_{0}$ ). Then, the wavelengths of the Stations 2, 3, 4 and 5 would be $\quad \lambda_{2}=1.08 \lambda_{0}, \quad \lambda_{3}=1.09 \lambda_{0}$, $\lambda_{4}=0.92 \lambda_{0}$ and $\lambda_{5}=0.9 \lambda_{0}$ respectively.

The lowest beamwidth among the stations would be $45^{\circ}$. The lowest $I / I_{0}$ ratio would be around 0.7 , a good value.

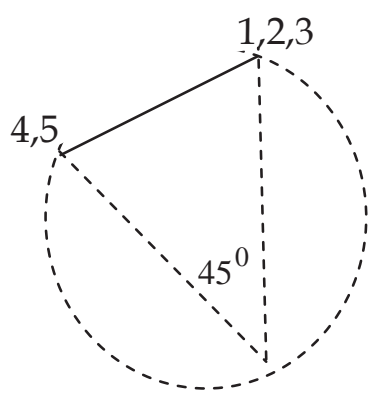

Figure 9 - Reception area for the Group 2

Outside the $45^{\circ}$ arc (beyond Beralapanahara), a good reception of all 5 stations can be predicted in the down-south region (Matara district area).

\section{Conclusions}

Since the Yagi-Uda antenna is highly sensitive to changes in its dimensions [2], its reception characteristics are usually optimized to receive a single transmission. This is done by maximizing the directive gain and the front to back ratio [1]. In practical situations like in the reception of TV channels, a single antenna installed on the rooftop is very often used for the reception of 
multiple transmissions. Two or more Yagi arrays are rarely used due to practical problems associated with their installation.

In this study, the possibility of designing a suitable antenna to cover a maximum number of UHF channels was explored. Although high gains can be achieved by increasing the number of elements in an array, the reduction of the beamwidth can adversely affect the physical coverage of the antenna. Thus, a 4-element array had to be selected as a compromise. The methodology was developed on the assumption that the domestic antenna receives only the direct signal from the transmitter. The modification of the radiation pattern due to signal diffraction and other factors was neglected.

The antenna coverage for the Colombo district and for the down south region covering Matara district was satisfactory. Hill country was not considered here.

The results show that an antenna designed for one particular station among a group of transmitting stations, might not be capable of receiving the rest of the stations successfully. The design parameters of the antenna should be selected considering the criteria discussed in this paper in order to maximize the number of stations it can receive.

\section{References}

1. David K. Cheng. “Gain optimization for Yagi-Uda Arrays" IEEE Transactions on Antennas and Propagation, 1991, Vol. 33, No.3, pp. 42- 46.

2. Jason D. Lohn, William F. Kraus, Dereck S. Lindan and Silvano P. Colombano. "Evolutionary optimization of Yagi-Uda Antennas" Proc. of the fourth international conference on Evolvable Systems, Tokyo, 2001. October 3-5, pp.236-243.

3. Orfanidis, Sophocles J. "Electromagnetic Waves and Antennas" Rutgers University, USA, 25 Aug. 2013. Web.

4. C. A. Cheng and David K. Cheng. "Optimum element lengths for Yagi-Uda arrays" IEEE Transactions on Antennas and Propagation, 1975, Vol. Ap-23, No. 1, pp. 8 -15.

5. David K. Cheng and C. A. Cheng. "Optimum element spacings for Yagi-Uda arrays" IEEE Transactions on Antennas and Propagation, 1973, Vol. AP-21, No.5, pp. 615-623.

6. Liang-Chi Shen. "Directivity and bandwidth of single-band and double-band Yagi arrays" IEEE Transactions on Antennas and Propagation, 1973, Vol.20, No.6, pp. $778-780$. 